\title{
Lingua e dialetto nella coscienza di Zeno
}

\section{Giovanni Albertocchi}

Universitat de Girona

\begin{abstract}
Trieste, città di frontiera, si riflette sulla scrittura di Italo Svevo, attraverso il doppio registro di lingua-dialetto. Ma nello scrittore, quello che potrebbe essere il tipico dualismo di tanti nostri letterati, acquista una connotazione etica, nel senso che investe un'altra giurisdizione, quella della menzogna e della verità. È una delle diverse frontiere che Italo Svevo attraversa nel suo percorso narrativo, e che può aiutare a comprendere, tra le altre cose, il suo rapporto ambivalente con la psicanalisi e la sua concezione della finzione.
\end{abstract}

Parole chiave: frontiera, dialetto, lingua, verità, menzogna, psicoanalisi, Dottor S.

\section{Abstract}

The work of the Italian writer Svevo reflects the frontier city of Trieste through a double register of language and dialect. But what might be considered the typical dualism of many of our writers, acquires ethical connotations in relation to the sense of falsehood and truth that another authority may invest. It is one of the various frontiers that Svevo crosses by means of novelistic detachment, and it helps to compromise, among other things, his ambivalent relationship with the psychoanalytical concept of fiction.

Key words: frontier, dialect, language, truth, falsehood, psychoanalysis, Doctor S.

\section{Trieste, città di frontiera}

Nel 1861 mentre il resto dell'Italia si unisce in uno stato indipendente sotto la dinastia dei Savoia, Trieste rimane al di là dei confini nazionali, in mano, per usare il linguaggio dell'irredentismo, allo straniero, ossia all'impero austroungarico che vi mantiene la propria lingua, la propria amministrazione e naturalmente la propria capitale, Vienna. Italo Svevo, uno dei romanzieri italiani più significativi del novecento, nasce così all'estero (Trieste, 1861) e con dati anagrafici, Aron Hector Schmitz, che la dicono lunga sul carattere composito della sua identità. La città stessa è un crogiolo di razze, di religioni e di culture che si mescolano e convivono grazie a quella singolare posizione geografica. "Il suo incanto maggiore - dice il poeta Umberto Saba — stava nella sua varietà. 
Svoltare un angolo di strada voleva dire cambiare continente. C'era l'Italia e il desiderio dell'Italia, c'era l'Austria (mica poi tanto cattiva quanto si pensava), c'era l'Oriente, c'era il Levante coi suoi mercanti in fez rosso, e molte altre cose ancora». ${ }^{1}$ Una città, aggiunge ancora Umberto Saba, sostanzialmente «nevrotica», affascinata dalla molteplicità della sua anima ma anche affannata nella ricerca della propria identità. Non è un caso che fu proprio Trieste a spalancare le porte della penisola alla psicanalisi e sempre Trieste la città da cui autorevoli discepoli di Sigmund Freud, in primo luogo Edoardo Weiss, cominciarono a diffondere il verbo del «maestro».

Giorgio Voghera ne Gli anni della psicanalisi racconta come intorno al Weiss si fosse creato una sorta di club di fanatici della nuova scienza che si scambiavano in continuazione "racconti ed interpretazioni di sogni e di lapsus» ${ }^{2}$ come se si trattasse di un gioco da salotto. Era un "continuo diagnosticare da dilettanti le proprie e le altrui neurosi, cercando di inquadrarle nell'una o nell'altra delle tre "fasi" (orale, anale, genitale, come si diceva allora) distinte da Freud; un continuo incolpare l'"es" degli altri — ma sotto sotto anche l'io delle più brutte intenzioni e dei più turpi sentimenti». ${ }^{3} \mathrm{Il}$ povero Edoardo Weiss interpellato continuamente «nelle condizioni e nelle ore più inopportune», si dava un gran da fare, inutilmente, per spiegare che l'analisi aveva le sue regole ben precise. Pare che stanco della pressione creatasi intorno a lui decidesse un bel giorno di lasciare Trieste per rifugiarsi in una città meno «nevrotica» come Roma.

Anche sul piano linguistico Trieste confermava la sua promiscuità: accanto al tedesco dell'amministrazione c'erano le lingue delle diverse comunità, l'italiano, il dialetto triestino, lo sloveno, il greco, l'yiddish, ecc. Ecco come descrive Roberto «Bobi» Bazlen la «delicata» geografia linguistica della città:

una città che parla un dialetto veneto, circondata da una campagna nella quale non si parla che una lingua slava, la parte più intellettuale della borghesia, che si sente staccata dal paese cui crede di appartenere per lingua e per cultura (benché non conoscano il «toscano» e benché la cultura [...] ma della cultura non parliamo), e che è dunque costretta, in pieno ventesimo secolo, a ricorrere a un frasario rettorico ottocentesco da Risorgimento, che tiene alta la fiaccola, che crede che l'italiano sia l'idioma gentil sonante e puro, e Firenze la città dei fiori $[\ldots]^{4}$

Trattandosi di Italo Svevo, cioè di uno scrittore italiano nato, diciamo così, in periferia, dovremo limitarci al dualismo così tipico nella nostra cultura fra lingua nazionale e dialetto. Non bisogna dimenticare che il nuovo Regno d'Italia nasceva, nel 1861, con quella famosa «asignatura pendiente» che imponeva alle nostre autorità, dopo aver fatto l'Italia di fare anche gli Italiani e

1. Cfr. Umberto Saba, "Trieste come la vide, un tempo, Saba», in Prose, Milano: Mondadori, 1964 , p. $215-216$.

2. Giorgio Voghera, Gli anni della psicanalisi, Pordenone: Edizioni Studio Tesi, 1980, p. 9.

3. Ibidem.

4. Roberto BAZLEN, Intervista su Trieste in Scritti. Milano: Adelphi, 1984, p. 246-247. 
soprattutto la lingua con cui potessero comunicare fra di loro. L'anno successivo alla proclamazione del Regno, il ministro della Pubblica Istruzione Emilio Broglio affidava ad Alessandro Manzoni l'incarico di elaborare un progetto di unificazione linguistica che avrebbe tardato comunque quasi un secolo prima di imporsi e non di certo non nei modi previsti dall'autore dei Promessi Sposi.

Lontano dai grandi centri culturali, Italo Svevo cerca di affinare come può la lingua in cui raccontare le storie dei suoi «inetti»: da buon provinciale sogna anche di recarsi a Firenze, magari con la scusa del viaggio di nozze, per «sciacquare» pure lui in Arno i "panni» dimessi della propria lingua come aveva fatto nel lontano 1827 Alessandro Manzoni accorso a Firenze per realizzare il famoso «bucato" linguistico del romanzo. Pare che il primo regalo di Hector alla moglie Livia Veneziani, sul finire del secolo, fosse proprio una copia dei Promessi Sposi, forse come surrogato sentimentale della «luna di miele» che i due non avevano potuto fare, come voleva la tradizione, a Firenze. Al di là dell'aneddoto, Svevo e Manzoni, come del resto molti altri scrittori non toscani avevano in comune la contraffazione linguistica a cui dovevano sottoporre il proprio universo letterario. Prendiamo i due "promessi sposi», Renzo e Lucia: dopo quasi due secoli ci siamo ormai abituati a sentirli parlare in toscano, anche se la «versione originale» della loro storia e soprattutto dei loro sentimenti si svolgeva in dialetto, per l'esattezza il lombardo della prima metà del seicento. Manzoni ha per così dire "doppiato" i due giovani e gli altri personaggi del romanzo in modo che tutti i lettori italiani potessero seguire le loro vicende.

Anche Italo Svevo si trova nella necessità di «doppiare» in lingua, personaggi che come lui si esprimevano abitualmente in dialetto. Per farlo non può ricorrere al toscano di cui ha soltanto una conoscenza approssimativa; si serve quindi di un suo italiano messo a punto in quel laboratorio di periferia, sobrio e preciso come se fosse tradotto da una lingua straniera. Non bisogna inoltre dimenticare che La coscienza di Zeno, nella finzione voluta dall'autore, è uno scartafaccio di memorie che il protagonista, Zeno Cosini, scrive per consiglio di uno psicanalista, il non meglio identificato Dottor $S$, come "preludio" alla terapia vera e propria. È probabile che entrambi, medico e paziente, pensassero che il lavoro di scavo sull'inconscio desse miglior risultati se fatto in italiano. $\mathrm{Ci}$ sarebbe anche l'ipotesi che il medico non fosse triestino: qualche studioso ha suggerito che dietro quella " $S$ » misteriosa si celasse nientemeno che Sigmund Freud oppure un suo discepolo, Wilhelm Stekel che aveva la curiosa abitudine di spifferare i segreti dei suoi pazienti. Fatto sta che per facilitare le cose al proprio analista, Zeno si rivolge a lui in lingua, il che darà origine, come vedremo in seguito, a gravi problemi, diciamo così, di comunicazione.

\section{nel suo puro dialetto triestino}

Pur esprimendosi in italiano Zeno lascia trapelare qua e là cenni di diglossia che era come si è detto la prassi linguistica della comunità italiana nella Trieste della prima metà del novecento. In questo modo ci avverte che sotto la 
superficie italiana del romanzo esiste uno strato dialettale in cui si muovono le voci «originali» di certi personaggi. Se non ce lo dicesse lui, che controlla dalla I ${ }^{a}$ persona l'intero spazio narrativo, non avremmo la possibilità di rendercercene conto. Una delle voci dialettali «doppiate» in italiano da Zeno, è quella di Giovanna, l'infermiera della clinica in cui Zeno si fa ricoverare per liberarsi dal vizio del fumo. "L'ultima sigaretta» è uno dei tanti propositi che la fragile volontà del protagonista non è riuscito a mantenere. Dopo aver provato di tutto, persino una terapia a base di scariche elettriche con il rocchetto di Ruhmkorff, decide, su consiglio della moglie Augusta, di rinchiudersi in una clinica. Qui, controllato a vista come un carcerato, spera di non cadere più nella tentazione. Una volta dentro invece il vizio si riaffaccia in tutta la sua intensità. Cerca allora di circuire l'infermiera, Giovanna, invitandola nella propria stanza a bere un bicchierino di cognac. I brindisi si susseguono mentre la donna, che ha ormai perduto il controllo, chiacchiera a ruota libera: «Non saprei ripetere esattamente quello ch'essa mi disse, dopo aver ingoiati vari bicchierini, nel suo puro dialetto triestino, ma ebbi tutta l'impressione di trovarmi da canto una persona che, se non fossi stato stornato dalle mie preoccupazioni, avrei potuto stare a sentire con diletto». ${ }^{5}$ Per convincerla a dargli da fumare, Zeno le fa credere che le sigarette eccitano la sua libido: «Bisognava sapere che quando io arrivavo a fumare una decina di sigarette diventavo terribile. Qualunque donna allora mi fosse stata a tiro si trovava in pericolo». ${ }^{6}$ Giovanna, vedova da diversi anni, sembra interessata all'esperimento; si informa addirittura sui tempi necessari perché il fumo faccia i suoi effetti. «Per dieci sigarette - precisa astutamente Zeno- io abbisogno di un'ora circa. Poi per aspettarne il pieno effetto occorre un'altra ora circa, dieci minuti di più, dieci di meno». ${ }^{7}$ L'infermiera lo squadra con un' «occhiataccia» assai significativa. Poi esce dalla camera dimenticandosi di chiudere la porta a chiave secondo le regole carcerarie della clinica. Dopo qualche istante piomba in mezzo alla camera un pacchetto con undici sigarette: «Per essere sicura la povera Giovanna aveva voluto abbondare». ${ }^{8}$ Zeno può finalmente riassaporare il fumo della sigaretta e subito dopo fuggire "da quella casa ove Giovanna aspettava il suo compenso».

La scena, benché "tradotta" in italiano, ha uno squisito sapore dialettale. È lo stesso protagonista infatti ad avvertirci che il cognac ha risvegliato, oltre ai sensi, anche la foga dialettale dell'infermiera che si esprime «nel suo puro dialetto triestino". Possiamo ipotizzare che lo stesso facesse Zeno per agevolare, con quella complicità linguistica, la sua opera di seduzione.

5. Per le citazioni ci siamo serviti di Italo Svevo, La coscienza di Zeno in Romanzi. (a cura di M. LaVAGETTO), Torino-Parigi: Einaudi-Gallimard, 1993. Op. cit., p. 528.

6. Op. cit., p. 530.

7. Op. cit., p. 531.

8. Ibidem.

9. Op. cit., p. 531 . 


\section{in lingua o dialetto?}

Un altro luogo del romanzo in cui l'autore, attraverso il solito portavoce, suggerisce una situazione di diglossia è quello in cui Zeno decide di recarsi da Giovanni Malfenti per chiedergli la mano della figlia Ada. Dopo la morte del padre, Zeno è alla ricerca di qualcuno che dia un senso ed un po' di ordine alla sua vita: il matrimonio, tra l'altro, è la scusa per rimanere nell'orbita di questo solido commerciante che gli fa ormai da «secondo padre» e che sembra averlo adottato non solo come figlio ma anche come socio negli affari. Zeno inizia così a frequentare la casa di Malfenti dove conosce le tre ragazze che potrebbero essere le possibili candidate: Ada, Augusta ed Alberta. Quella coincidenza alfabetica suggerisce a lui che si chiama Zeno «il sentimento che stessi per prendere moglie lontano dal mio paese». ${ }^{10}$ Zeno è affascinato dalla più bella delle sorelle, Ada, anche se questa non gli da' molte speranze, anzi gli suggerisce di rivolgere le sue attenzioni ad Augusta. Sapremo in seguito che Ada è innamorata di un altro pretendente, il bel Guido Speier. Zeno comunque non demorde e pensa di chiederla in moglie a Giovanni. Mentre si dirige all'ufficio del Malfenti, fra i tanti dubbi che gli passano per la mente ce n'è anche uno di natura linguistica: «Mi preoccupava tuttavia la quistione se in un'occasione simile avrei dovuto parlare in lingua o dialetto». ${ }^{11}$ Questa è purtroppo una curiosità che non vedremo mai soddisfatta perché Giovanni Malfenti era appena uscito dall'ufficio per andare al Tergesteo, la borsa di Trieste, e la prosecuzione delle trattative matrimoniali che si concluderanno con le nozze con Augusta, avranno un'altra ben più abile intermediaria nella futura suocera, la signora Malfenti.

Il dubbio linguistico di Zeno sembra anche presagire l'esito incerto dell'operazione, visto che Ada è già stata destinata all'altro pretendente. Quella richiesta in matrimonio non è quindi cosa da prendere alla leggera: Zeno si chiede se non sia il caso di ricorrere ad un protocollo espressivo più autorevole di quello che è da supporre intercorresse normalmente fra i due e rivolgersi al futuro suocero in bell'italiano.

\section{condannati al nostro dialettaccio}

Poco tempo dopo, in occasione di un ricevimento in casa Malfenti, Zeno conosce Guido Speier e deve ammettere amaramente che sarà difficile spuntarla su un rivale così agguerrito. Guido è un giovane affascinante con dei bellissimi capelli «bruni e un po' ricciuti» che «coprivano tutto lo spazio che madre natura aveva loro destinato, mentre molta parte della mia testa - deve ammettere desolato Zeno - era stata invasa dalla fronte». ${ }^{12}$ Come se non bastasse, Guido ha enormi risorse anche sul piano espressivo: "parlava toscano con grande naturalezza mentre io e Ada eravamo condannati al nostro dialettaccio». ${ }^{13}$ 
L 'italiano che parla Guido non è quello approssimativo usato dai triestini come Zeno quando mettono da parte il dialetto: è il modello linguistico della grande tradizione, quello che Alessandro Manzoni ha investito ufficialmente nel 1868 con il rango di lingua nazionale. Guido, oltre alla bellezza, i soldi, la grande maestria nel suonare il violino, si presenta in quella frangia asburgica della penisola «unto» dell'idioma antico della patria lontana. Il povero Zeno, afflitto da calvizie incipiente e dall'handicap dialettale, ha soltanto dalla sua un gran senso dello humour che Ada però mostra di non apprezzare. Guido e Ada coroneranno di lì a poco il loro sogno d'amore, mentre Zeno dovrà ripiegare sulla sorella bruttina, Augusta. Con il matrimonio, il giovane, oltre a "sistemarsi» come desiderava da quando era morto suo padre, potrà avere sempre sotto tiro i due responsabili della sua sconfitta con i quali manterrà un eccezionale rapporto di ambiguità. Siamo ormai negli strati leggendari della coscienza di Zeno ove regna l'ambivalenza e da cui arrivano segnali, volutamente ignorati dall'interessato, che dimostrano come sia sempre innamorato di Ada e come, nonostante gli sforzi non ce la faccia proprio ad affezionarsi al cognato. Solo dopo la sua morte Zeno dovrà riconoscere di «aver sempre odiato Guido e di essergli stato accanto, assiduo, in attesa di poter colpirlo». ${ }^{14}$

\section{glielo traducevo in triestino}

La vita matrimoniale di Zeno procede intanto a gonfie vele. Anche l'adulterio è incorporato nelle regole borghesi della vita di coppia che richiede, nella mentalità del nostro personaggio, qualche accorgimento per tenere a bada la routine. Capita così a Zeno di conoscere una procace giovinetta, Carla Gerco da cui si reca per ravvivare l'eros coniugale. Soltanto in questo modo è possibile recepire la singolare affermazione secondo la quale «lì accanto a Carla, rinacque intera la mia passione per Augusta». ${ }^{15}$

Carla Gerco studia, con scarsi risultati, canto, il che offre a Zeno la possibilità di mascherare la scappatella sotto l'alibi filantropico di aiutare una fanciulla bisognosa. Il primo regalo che le fa è il "Trattato completo dell'arte del Canto (Scuola di Garcia) di E. Garcia (figlio) contenente una relazione sulla Memoria riguardante la Voce Umana presentata all'Accademia delle Scienze di Parigi». ${ }^{16}$ Mario Lavagetto, nella sua documentatissima edizione del romanzo di Svevo, ci avverte che il libro "destinato a fare da Galeotto" è in realtà il Traité complet de l'art du chant di Manuel Patricio Rodriguez Garcia (sic), edito a Parigi nel $1840 .{ }^{17} \mathrm{E}$ quindi un libro francese, il che giustifica il fatto che Zeno, convinto che tale metodo «le avrebbe insegnato il modo di rendere le note solide come il metallo e dolci come l'aria» ${ }^{18}$ decida in primo luogo di leg- 
gerglielo traducendolo via via in italiano. E poi, una volta tradotto, di spiegarglielo sempre in italiano. Infine "quando non bastava, glielo traducevo in triestino». Si tratta, come si vede di una complessa strategia in cui Zeno sfoggia, con scarsi risultati visto che «essa non sentiva moversi niente nella sua gola», tre diverse competenze linguistiche: francese, italiano e triestino. Ma l'obbiettivo didattico è assolutamente secondario: ciò che preme a Zeno è fornire alla propria coscienza fedifraga un alibi sufficiente a smaltire gli inevitabili sensi di colpa. Mentre lo vediamo in azione come ambiguo pedagogo che cerca in realtà di circuire la fanciulla, osserviamo ancora una volta l'indizio linguistico che trapela dalla scena. L'italiano in cui traduce «le teorie del Garcia» è solo il primo passaggio comunicativo che deve poi sfociare necessariamente nel dialetto, strumento didattico assai più convincente da cui Zeno «finge» di riproporsi migliori risultati, ma che è anche probabilmente, come già era avvenuto con Giovanna, il registro espressivo dell'incipiente seduzione. Zeno, in fondo, tradisce la moglie in dialetto triestino.

Il dialetto alla fine prenderà il sopravvento anche sul metodo "del Garcia» e Carla si deciderà ad offrire all'amante qualche prova più alla mano del suo repertorio musicale cantandogli una maliziosa canzonetta triestina:

Fazzo l'amor xe vero

Cossa ghe xe de mal

Volè che a sedes'ani

Stiò là come un cocal $[\ldots]^{19}$

La complicità dialettale spalanca le porte della tenerezza e del desiderio: Zeno, «per la prima volta» contempla la bellezza di Carla: «un ovale purissimo interrotto dalla profonda e arcuata incavatura degli occhi e degli zigomi tenui, reso anche più puro da un biancore niveo». ${ }^{20}$ Forse per la prima volta la desidera come donna che gli offre, in dialetto, la giovanile sensualità di «quella carne che pareva trasparente, e celava tanto bene il sangue e le vene forse troppo deboli per poter apparire». ${ }^{21}$ Anche su Carla la canzone triestina ha un effetto liberatorio: "Quando terminò e mi trovò in piena ammirazione, anch'essa per la prima volta oltre che amarmi mi volle veramente bene». ${ }^{22}$ Il dialetto, più che il libro "del Garcia» è quindi il vero "Galeotto" fra la robusta sensualità di Carla e quella un po'impacciata di Zeno.

\section{con ogni nostra parola toscana noi mentiamo!}

I quattro prelievi realizzati nel sottosuolo linguistico del romanzo confermano la presenza dello strato dialettale, al punto che si potrebbe ipotizzare per

19. «Faccio l'amore è vero / cosa c'è di male? / Volete cha a sedici anni / stia lì come una scema.?» Op. cit., p. 716.

20. Op. cit., p. 716.

21. Ibidem.

22. Op. cit., p. 717. 
La coscienza di Zeno la condizione di romanzo bilingue: scritto in lingua italiana ma "parlato" in dialetto triestino. Visto che noi lettori abbiamo accesso soltanto a uno dei registri dell'opera, Svevo ci invia periodicamente segnali sull'esistenza di quell'altro che Angelo Ara e Claudio Magris hanno definito il "grembo edipico e sotterraneo del dialetto». ${ }^{23}$

Verso la fine del romanzo però la normale condizione di bilinguismo su cui lo scrittore è venuto via via informandoci, viene bruscamente alterata. Lingua e dialetto sono improvvisamente investiti di prerogative che non riguardano la diglossia ma l'etica e la credibilità dello scrittore. Ci troviamo nel capitolo finale del romanzo intitolato Psico-analisi. Zeno sta scrivendo, come si ricorderà le sue memorie come strumento di appoggio alla psicanalisi vera e propria che, ci dice, ha praticato per «sei mesi interi». Ma ora, confessa, non ne può più né di quella presunta terapia buona soltanto «per commuovere qualche vecchia donna isterica» ${ }^{24}$ né del Dottor $S$. che con freudiana prosopopea gli ha affibbiato la malattia "diagnosticata a suo tempo dal defunto Sofocle sul povero Edipo: Avevo amata mia madre e avrei voluto ammazzare mio padre». ${ }^{25}$ "Chi di psico-analisi s'intende», direbbe il Dottor S., sa dove piazzare tutta questa antipatia. Fatto sta che l'insofferenza di Zeno è tale che decide di interrompere la cura e la redazione delle memorie. Per inciso va detto che tale decisione manderà su tutte le furie il Dottor $S$. il quale per vendicarsi le pubblicherà. Ecco perché, stando alle regole del gioco fissate dallo scrittore, noi ora le possiamo leggere in un libro stampato.

Torniamo al capitolo ottavo con le confessioni di Zeno ancora sul tavolino del suo studio. Per farsi beffe dello psicanalista e della sua cura dice che quanto ha scritto finora potrebbe non corrispondere alla verità. Il fatto è, confessa candidamente, che quelli come lui che si esprimono abitualmente in dialetto suppliscono con la menzogna o la reticenza alla scarsa conoscenza dell'italiano, selezionando solo ciò che sono in grado di dire senza ricorrere al vocabolario:

Egli [il Dottor S.] non studiò che la medicina e perció ignora che cosa significhi scrivere in italiano per noi che parliamo e non sappiamo scrivere il dialetto. Una confessione in iscritto è sempre menzognera. Con ogni nostra parola toscana noi mentiamo! Se egli sapesse come raccontiamo con predilezione tutte le cose per le quali abbiamo pronta la frase e come evitiamo quelle che ci obbligherebbero di ricorrere al vocabolario! È proprio così che scegliamo dalla nostra vita gli episodi da notarsi. Si capisce come la nostra vita avrebbe tutt'altro aspetto se fosse detta nel nostro dialetto. ${ }^{26}$

In base alle provocatorie parole di Zeno, la diglossia non sarebbe più solo una condizione linguistica, ma etica, nel senso che non esprimerebbe più sol-

23. Angelo Ara, Claudio Magris, Trieste. Un’identità di frontiera, Torino: Einaudi, 1987, p. 134.

24. Op. cit., p. 865.

25. Op. cit., p. 865.

26. Op. cit., p. 866. 
tanto la alternativa fra lingua e dialetto ma anche tra menzogna e verità. In parole povere, Zeno dichiara di mentire quando si esprime in italiano e di dire la verità solo quando usa il dialetto. Se pensiamo che il libro è scritto interamente in italiano, possiamo subito immaginare la ripercussione che le sue parole avranno sulla credibilità di ciò che ha raccontato. Il primo a risentirne è il Dottor S., che su quelle menzogne ha impostato una terapia ed ha formulato una diagnosi. Ma dietro di lui ci siamo anche noi lettori che su quelle affermazioni rilasciate via via dal protagonista ci siamo fatti delle idee che speravamo di veder confermate nell'epilogo. Il Dottor S., a pensarci bene, aveva messo in guardia già nella "prefazione", che è l'unico spazio del romanzo, come ha ben rilevato Eduardo Saccone ${ }^{27}$ in cui ci è dato di ascoltare una voce che non sia quella di Zeno, sulle «tante verità e bugie ch'egli [Zeno] ha qui accumulate! $\left[\ldots . .{ }^{28}{ }^{28}\right.$ Pensavamo fosse solo lo sfogo di uno psicanalista beffato dal suo paziente invece era probabilmente lo stesso Svevo che cercava di stipulare con noi questo singolare "patto narrativo» ${ }^{29}$ in modo che le dichiarazioni sul «bilinguismo" di Zeno non ci sorprendessero più di tanto.

Che il rapporto tra lingua e dialetto, sul piano espressivo, non fosse tutto rose e fiori, ce lo aveva lo aveva già dimostrato Alessandro Manzoni affermando, nella «Seconda introduzione» al Fermo e Lucia che "quando l'uomo che parla abitualmente un dialetto si pone a scrivere in una lingua, il dialetto di cui egli s'è servito nelle occasioni più attive della vita, per l'espressione più immediata e spontanea dei suoi sentimenti, gli si affaccia da tutte le parti, s'attacca alle sue idee, se ne impadronisce [...]; gli cola dalla penna e se egli non ha fatto uno studio particolare della lingua, farà il fondo del suo scritto». ${ }^{30}$ Qui però, i depositi che turbano la pagina di Zeno non sono quelli dialettali: è la lingua italiana che "cola» sgradevolmente dalla sua penna lasciando sulla pagina una scia interminabile di menzogne.

\section{Una confessione in italiano non poteva essere né completa né sincera}

Subito dopo aver individuato il meccanismo di selezione espressiva che riduce, in italiano, la quota di sincerità incrementando, al contrario, la menzogna, Svevo ce ne offre una dimostrazione pratica. L'occasione è data dalle perplessità del Dottor S. riguardo alla veridicità delle informazioni che Zeno fornisce via via sul cognato, Guido Speier. In effetti costui, dopo la brillante apparizione del capitolo quinto (La storia del mio matrimonio) in cui conquista definitivamente il cuore di Ada, subisce successivamente un degrado progressivo della sua immagine fino al suicidio, programmato, come al solito, per spillare quattrini alla consorte, con la differenza che questa volta, nonostante le precauzio-

27. Eduardo SACCONE, Commento a Zeno, Bologna: il Mulino, 1991.

28. Op. cit., p. 509.

29. Vedi a questo proposito: Giulio SAVELLI, «Il dottor S. e la storia virtuale di Zeno», Strumenti critici, XI, 1 (gennaio 1996), 93-100.

30. Alessandro MAnZONI, Fermo e Lucia. Vol.1. Torino: Einaudi, 1971, p. 5. 
ni, nessuno lo viene a salvare e per lui è veramente la fine. Certo, la nostra unica fonte di informazione è Zeno, ma, nonostante l'antipatia che nutre nei confronti del cognato, nessuno di noi si sognerebbe mai di metterne in dubbio le parole. Il Dottor $S$. invece non è di questo avviso. Insospettito forse da ciò che ha annusato nell' inconscio del paziente si trasforma in detective e cerca di ristabilire la verità. È il primo psicanalista, a quanto ci risulta, che indaga sui propri pazienti. Viene così a scoprire che «un grandioso deposito di legnami, vicinissimo alla casa dove noi pratichiamo la psico-analisi, era appartenuto alla ditta Guido Speier \& C.» ${ }^{31}$ Il povero Guido non era quindi quel cialtrone miserabile e buono a nulla come l'aveva dipinto Zeno. Era riuscito ad avviare una prospera ditta di legnami e forse era titolare di altre attività. Il Dottore chiede a Zeno perché non gliene ha mai parlato ed eccone la risposta:

Se ne avessi parlato sarebbe stata una nuova difficoltà nella mia esposizione già tanto difficile. Quest'eliminazione non è che la prova che una confessione fatta da me in italiano non poteva essere né completa né sincera. In un deposito di legnami ci sono varietà enormi di qualità che noi a Trieste appelliamo con termini barbari presi dal dialetto, dal croato, dal tedesco e qualche volta persino dal francese [...]. Chi m'avrebbe fornito il vero vocabolario? Vecchio come sono avrei dovuto prendere un impiego da un commerciante in legnami toscano? 32

La reticenza di Zeno non è che l'applicazione di quel singolare statuto dei dialettofoni che raccontano "con predilezione» solo le cose per cui hanno "pronta la frase» mentre evitano quelle che li obbligherebbero a «ricorrere al vocabolario». In questo caso concreto, Zeno afferma che non essendo in grado di riferire in italiano i nomi dei legnami immagazzinati nel deposito della Ditta Guido Speier \& C., è costretto a censurare parte delle informazioni sul cognato pur con il rischio di alterare profondamente la verità. L'episodio di Guido imprenditore di legnami, lo avrebbe potuto raccontare soltanto dopo uno stage linguistico presso «un commerciante in legnami toscano». Quel famoso «bucato" manzoniano, vanto della tradizione ottocentesca, si sposterebbe così dalle rive dell'Arno ad una falegnameria dell'entroterra. L'impasse di Zeno davanti ai «termini barbari presi dal dialetto» ricorda quella analoga di Alessandro Manzoni davanti all'insegna del forno che stanno per assaltare nel cap. XII dei Promessi Sposi i rivoltosi milanesi: "Nella strada chiamata la Corsia de' Servi, c'era, e c'è tuttavia un forno, che conserva lo stesso nome; nome che in toscano viene a dire il forno delle grucce, e in milanese è composto di parole così eteroclite, così bisbetiche, così salvatiche, che l'alfabeto della lingua non ha i segni per indicarne il suono». ${ }^{33}$ Di fronte all' «ineffabilità» negativa del dialetto, Manzoni traduce «el prestin di scansc» ne «il forno delle grucce», mentre Zeno, con il legname, non ci prova neppure e se ne sta zitto. 


\section{Zeno, un "bugiardo autorizzato»}

L'episodio del magazzino di legnami apre una voragine nell'intera confessione di Zeno, in quanto lascia supporre una sequela ben più ampia di menzogne o di versioni distorte. Il primo sospetto che ci viene in mente è che abbia mentito anche su Ada, la donna di cui è innamorato e che lo ha sempre rifiutato. Ecco ciò che riserva ad essa la narrazione: prima un marito incompetente ed infedele che la ricatta, poi una brutta malattia, il morbo di Basedow che deforma il suo bellissimo volto. Ci sorge il dubbio che qui non sia l'incompetenza linguistica a modificare la versione dei fatti ma il rancore di Zeno che non ha dimenticato lo sgarbo subito dalla donna e che ora si vendica su di lei come un bambino stizzito facendola diventare brutta. Non è un caso che Zeno si interroghi periodicamente se sia buono o cattivo. Forse è un modo per avvertirci di stare in guardia e e di non prenderlo troppo sul serio. Come sapere se dice la verità? Se fosse ancora in vita il Dottor S., potremmo tentare di convincerlo a proseguire le indagini.

La menzogna non è soltanto il capriccio infantile di un nevrotico ma una strategia espressiva sofisticata. Vediamone i destinatari o se vogliamo le vittime. C’è in primo luogo lo psicanalista, perché è a lui che Zeno indirizza le sue confessioni. E poi ci saremmo noi lettori che accediamo al testo giacché il Dottor S. lo ha pubblicato "per vendetta». Diciamo allora che la menzogna si ripercuote su due zone della creazione rappresentate rispettivamente dal Dottor S. e dai lettori, cioè la psicanalisi come terapia ed il romanzo come genere letterario.

Veniamo alla psicanalisi: la censura di Zeno sul deposito dei legnami sancisce la possibilità che il paziente racconti allo psicanalista solo ciò che vuole, ossia una versione ridotta e distorta della verità. Se pensiamo che è attraverso l'ascolto che lo psicanalista interpreta e cura la sua nevrosi, ne deduciamo che le possibilità di successo della terapia sono assai limitate o perlomeno compromesse nel caso che il paziente sia, come il nostro Zeno, un bugiardo matricolato. La menzogna, la reticenza o il silenzio, sono d'altronde strategie che Sigmund Freud aveva già previsto e codificato come manifestazioni della «resistenza» che è, a suo avviso, una delle tante risorse negative del nevrotico che l'analista riesce a convertire in materiale produttivo. Da un punto di vista dell'ortodossia freudiana, l'atteggiamento di Zeno rientrerebbe quindi nella tipica «resistenza» del paziente, come conferma Mario Lavagetto nel suo saggio La cicatrice di Montaigne (Sulla bugia in letteratura). Secondo il critico Zeno è sì «un vecchio bugiardo che prende la parola e scrive a un destinatario preciso [il Dottor S.], con l'intento di circuirlo e di ingannarlo», ${ }^{34} \mathrm{ma}$ è soprattutto un nevrotico che «resiste» nei modi previsti all'analista che lo vuole stanare. Se invece diamo retta al personaggio e forse allo stesso autore, visto che i due hanno inevitabilmente molte cose in comune, vediamo che si tratta esclusivamente di una strategia creativa, consapevole, ordita dal paziente per portare 
fuori strada l'analista. Non dimentichiamo che nell'ultimo capitolo del romanzo il dottore è bollato come «bestione» incompetente che si lascia abbindolare con dei falsi sogni che Zeno improvvisa lì per lì per divertirsi alle sue spalle. Zeno sospetta addirittura che sia lui il vero nevrotico, l' 'istericone che per aver desiderata invano sua madre se ne vendica su chi non c'entra affatto».

Il nostro personaggio lancia le sue menzogne come ordigni esplosivi contro la credibilità della psicanalisi che esce qui a malpartito. Appare come una terapia fasulla costruita su «un patto narrativo» come qualsiasi procedura basata sulla finzione: è ancora Zeno a confermarci che «inventare è una creazione, non già di menzogna». Il Dottor $S$. potrebbe funzionare come destinatario del racconto di Zeno solo se rinunciasse al ruolo di terapeuta ed accettasse le confessioni per quello che sono, un semplice atto creativo frutto dell'invenzione. Zeno diventerebbe così un personaggio capace di suscitare la sua curiosità, non più un «malato» da curare.

Sul comportamento di Zeno nei confronti del Dottor S. ha certamente influito la vicenda biografica di Bruno Veneziani, cognato dello scrittore. Bruno, per il carattere irrequieto, l'omosessualità e l'uso della droga, aveva le carte in regola per diventare la "pecora nera» di quella operosa famiglia di industriali triestini arricchitasi con la produzione di vernici sottomarine. L'unico a seguire con curiosità e simpatia la sua carriera di «irregolare» era proprio Italo Svevo. La madre, l'energica Olga Veneziani, faceva di tutto per riportare Bruno sulla retta via. Nel 1911 si rivolse addirittura a Sigmund Freud in persona perché lo sottoponesse a quella nuova terapia di cui a Trieste si faceva un gran parlare. Freud ci provò ma con scarsi risultati: Bruno era un "osso duro» che non accettava il "tranfert» e si faceva per di più beffe del maestro al punto che costui lo mise alla porta come un alunno indisciplinato con le seguenti parole: «Io posso guarire chi desidera la guarigione, non chi la rifiuta». ${ }^{35}$ Qualche anno dopo, nel 1920, Olga Veneziani tornò alla carica: pregò l'amico Edoardo Weiss, discepolo, come già si è visto, di Sigmund Freud, di mettere una parola buona presso il maestro perché riprendesse in cura il figlio che continuava a combinarne di tutti i colori. Quando ricevette la lettera di Weiss, Freud dovette rabbrividire: dalla risposta si capisce che lui di quel tipo non voleva più sentirne parlare, consigliava addirittura di spedirlo lontano, in Sud America:

Due sono le cose che gli mancano: la prima, quel certo conflitto di sofferenza fra il suo Io e le sue pulsioni istintuali, perché in effetti è molto soddisfatto di sé e soffre soltanto per l'antagonismo delle condizioni ambientali; la seconda, quel tanto di normalità dell'Io che gli consente di collaborare con l'analista. Egli invece, tenterà sempre ad ingannarlo, a fingere, per levarselo di torno [il neretto è nostro]. Entrambi questi difetti convergono in uno solo, e cioè nello sviluppo di un Io estremamente narcisistico, refrattario ad ogni influenza, che sfortunatamente può far leva su tutti i suoi talenti e le sue doti personali. Penso, quindi, che al momento attuale un trattamento, da parte mia o di altri, non possa giovargli. Può darsi però, che riesca a recuperare da solo, come Mira- 
beau, al cui tipo probabilmente appartiene, e pur conservando tutti i suoi vizi riesca a conseguire qualche risultato degno di nota. Ma non è molto probabile. In tutto ciò non considero minimamente il fatto che sia omosessuale. Potrebbe continuare ad esserlo e, tuttavia, condurre vita ordinata e ragionevole. ${ }^{36}$

Freud suggeriva in un primo momento di mandarlo a Baden-Baden nella clinica di un analista "selvaggio", il dott. Georg Groddek, ${ }^{37}$ e poi concludeva dichiarando bruscamente che «Nella peggiore delle ipotesi, gente come il dottor $^{* *}$ [Bruno Veneziani] la si spedisce con un po' di denaro oltreoceano, per esempio in Sud America, lasciando che vi cerchi e trovi il proprio destino».

Quella lettera assai dura dovette dispiacere non poco alla famiglia Veneziani: anche Svevo lo venne a sapere, nonostante le cautele ed i silenzi dei suoceri su quel figlio scapestrato, e ne rimase molto impressionato. Accenni alla storia di quel cognato in continuo pellegrinaggio da uno psicanalista all'altro, ne troviamo ad esempio in un racconto autobiografico, Soggiorno londinese a proposito del modo in cui lo scrittore era entratto in contatto con la psicanalisi:

Un mio amico nevrotico corse a Vienna per intraprenderla. Lavviso dato a me fu l'unico buon effetto della sua cura. Si fece psicanalizzare per due anni e ritornò dalla cura addirittura distrutto: Abulico come prima ma con la sua bella abulia aggravata dalla convinzione ch'egli, essendo fatto così, non potesse agire altrimenti. È lui che mi diede la convinzione che fosse pericoloso di spiegare ad un uomo com'era fatto ed ogni volta che lo vedo lo amo per l'antica amicizia ma anche per la nuova gratitudine. ${ }^{38}$

Qui Bruno è, come si vede, un «amico» a cui Svevo dice di essere molto affezionato, il che fa intuire come lo avesse impressionato negativamente l'atteggiamento di Freud nei suoi confronti. Ad un conoscente, Valerio Jahier, che voleva sottoporsi ad una terapia psicanalitica, Svevo scrive il 10 dicembre 1927: «Grande uomo quel nostro Freud ma più per i romanzieri che per gli ammalati. Un mio congiunto uscì dalla cura durata varii anni addirittura distrutto». ${ }^{39}$ In un'altra lettera del 27 dicembre cerca di correggere il tiro in quanto teme di aver incrinato «la speranza» che Jahier «ripone nella cura», ma in sostanza riconferma la sua idea assai negativa: «Certo è ch'io non posso men-

36. Op. cit., p. 50.

37. Bruno Veneziani andrà realmente a Baden-Baden, nel giugno 1921, accompagnato dalla madre e dall'amico-cognato Ettore Schmitz ma neppure stavolta la cura darà dei risultati. Lo racconta Fulvio Anzellotti, pronipote di Svevo, in Il segreto di Svevo. Pordenone: Studio Tesi, 1985. "Georg Groddeck —afferma l'Anzellotti- parla di Bruno anche nel suo famoso Libro dell'Es, e lo classifica fra i "figli della balia". Per l'“analista selvaggio" ogni bambino allattato da una balia è collocato in una situazione di incertezza e di dubbio che egli non abbandonerà mai più, combattuto come è tra l'affetto verso la vera madre, e quello verso l'altra creatura al cui seno si attacca ogni giorno, di cui succhia il latte, scaldandosi al tepore della sua pelle e respirandone l'odore.» (p. 153).

38. Italo Svevo, Racconti, saggi, pagine sparse, Milano: dall'Oglio, 1969, p. 688.

39. Italo Svevo, Epistolario. Milano: dall'Oglio, 1966, p. 857-858. 
tire e debbo confermarle che in un caso trattato dal Freud in persona non si ebbe alcun risultato. Per esattezza debbo aggiungere che il Freud stesso, dopo anni di cure implicanti gravi spese, congedò il paziente dichiarandolo inguaribile. Anzi io ammiro il Freud, ma quel verdetto dopo tanta vita perduta mi lasciò un'impressione disgustosa». ${ }^{40}$

Prima di tornare a Zeno, diciamo per chi volesse saperne di più su Bruno Veneziani che la sua storia ebbe, nonostante tutto, un «lieto fine». «La sua guarigione - racconta Fulvio Anzellotti- arrivò per Bruno sotto le sembianze di un bel marinaio» ${ }^{41}$ conosciuto a Roma e con cui vivrà fino alla morte avvenuta nel 1952.

Di fronte al Dottor S., Zeno rappresenterebbe quindi un copione scritto a due mani da Italo Svevo e da Bruno Veneziani, per vendicarsi di tutto il tempo perso da quest' ultimo andando su e giù fra Trieste e Vienna e soprattutto di quella lunga lotta sostenuta contro di lui dai migliori specialisti europei, Freud in testa, per guarirlo da una "malattia» a giudizio di Svevo inesistente. Ecco quindi dove conduce la perversa strategia di Zeno: a smontare la credibilità della psicanalisi visto che poggia su un sistema di lacune intenzionali e di bugie. Dietro il Dottor $S$. che riceve in pieno viso, come i personaggi del cinema muto, la torta-sberleffo del suo paziente, possiamo quindi immaginare la presenza di un'intera dinastia di psicanalisti, a cominciare dal capostipite, Sigmund Freud ed includendovi pure Edoardo Weiss che, pur senza volerlo, viveva come si è visto, con i suoi pazienti una sorta di frenetica promiscuità in cui poteva maturare un gesto, così poco deontologico, come quello compiuto dall'analista di Zeno di rivelare i segreti del suo paziente.

Nella lettera già citata a Valerio Jahier del 10 dicembre 1927 c'è però un'interessante precisazione dello scrittore riguardo al fondatore della psicanalisi: «Grande uomo quel nostro Freud ma più per i romanzieri che per gli ammalati». Il Freud messo simbolicamente alla berlina da Zeno è solo quello che vuole guarire gli ammalati. Insomma è solo l'aspetto terapeutico della nuova scienza che si vuole mettere in discussione, non quello scientifico che ha somministrato all'umanità e quindi anche ai romanzieri eccezionali strumenti di analisi e grandi opportunità narrative.

Sul piano più propriamente letterario, le menzogne di Zeno risultano invece del tutto inoffensive, anzi, si potrebbe dire, assai raccomandabili in quanto sono in perfetta sintonia con le leggi di un sistema che si basa sulla finzione. $\grave{E}$ lo stesso Zeno a sostenere, come si è già detto, che «inventare è una creazione, non già una menzogna». In un saggio intitolato, guarda caso, La verdad de las mentiras, lo scrittore peruviano Mario Vargas Llosa, da anni impegnato in una sua personale crociata a favore della menzogna in letteratura, afferma che «la novela es un género amoral, o más bien, de una ética sui generis, para la cual verdad o mentira son conceptos exclusivamente estéticos». ${ }^{42}$ 
Cesare Segre conferma che fra le diverse competenze dello scrittore c'è anche il diritto ad una «selezione di carattere funzionale»: proprio quella eseguita da Zeno, in qualità di autore delle proprie memorie, allorché scarta l'informazione sul magazzino di legnami che potrebbe compromettere l'immagine ambigua e negativa che ci vuole trasmettere di Guido. Lo scrittore, sempre secondo Segre, è in fondo un «bugiardo autorizzato» ${ }^{43}$ e come tale si comporta appunto Zeno che mente con cognizione di causa. Dietro le quinte del romanzo Svevo ha l'aria di essere dello stesso avviso e lo dimostra giocando, si fa per dire, a carte scoperte. Riparandosi dietro la curiosità del Dottor $S$., fa sì che la famigerata «menzogna dei legnami» riemerga dalla confessione lacunosa di Zeno per essere ufficialmente reintegrata nello status legittimo di variante narrativa. 